\title{
Nonlinear Sampled-Data Stabilization of Dynamically Positioned Ships
}

\author{
Hitoshi Katayama
}

\begin{abstract}
In this brief, we consider the nonlinear sampled-data stabilization of dynamically positioned ships. Using the nonlinear sampled-data control theory developed by Nesic et al. and the integrator backstepping technique for the Euler approximate model, we design semiglobally practically asymptotically (SPA) stabilizing controllers. We design both state feedback and output feedback controllers. We give a numerical example to illustrate the design methods.
\end{abstract}

Index Terms-Control of ships, Euler approximate model, nonlinear sampled-data control, stabilization.

\section{INTRODUCTION}

$\mathbf{F}$ ROM THE beginning of the 20th century, the design of control systems for ships has been actively considered. Propotional-integral-derivative (PID) control, linear quadratic optimal control, Kalman filtering, $\mathrm{H}_{\infty}$ control, sliding mode control, feedback linearization, and etc., have been applied to design control systems (for details see [3], [13], and references therein). Recently, nonlinear backstepping design techniques [3], [14], the control problems of underactuated dynamic positioning [15], and the nonlinear observer design technique [7] for ships have been also discussed. The analysis and synthesis of the control problems for ships have been considered based on linear or nonlinear continuous-time models and the design methods of continuous-time controllers have been mainly discussed.

Practical and modern control systems usually use digital computers as discrete-time controllers with samplers [analog-to-digital (A/D) converters] and zero-order holds [digital-to-analog (D/A) converters] to control continuous-time systems [2]. Such a control system involves both continuous-time and discretetime signals in a continuous-time framework and is called a sampled-data system. For linear systems, the sampled-data control theory has been widely studied (for details see [2] and references therein). On the other hand, due to the difficulty to find equivalent and useful discrete-time models of sampled-data nonlinear systems, the sampled-data control theory for nonlinear systems has not been developed compared to linear systems. But recently, the framework to design controllers for nonlinear sampled-data systems based on discrete-time approximate models is proposed [6], [11], [12]. Several design methods such as an emulation and controller redesigns of continuous-time controllers [8], an integrator backstepping for discrete-time nonlinear systems [10] and controller designs by receding horizon methods

Manuscript received October 28, 2007; revised January 20, 2009. Manuscript received in final form February 01, 2009. First published May 19, 2009; current version published February 24, 2010. Recommended by Associate Editor L. Villani.

The author is with the Department of Electrical and Electronic Engineering, Shizuoka University, Hamamatsu 432 8561, Japan (e-mail: thkatay@ ipc.shizuoka.ac.jp).

Digital Object Identifier 10.1109/TCST.2009.2014876
[9] have been proposed to guarantee the stability of nonlinear sampled-data systems. Moreover, the design of nonlinear observers for sampled-data nonlinear systems has been considered based on both discrete-time approximate models and continuous-time models [1]. But as mentioned in [6], case studies and practical implementations of the controllers designed in the framework of the nonlinear sampled-data systems have not been discussed in the literatures.

In this brief we consider the design of semiglobally practically asymptotically (SPA) stabilizing controllers for dynamically positioned ships in this framework. We first design state feedback SPA stabilizing controllers by extending an integrator backstepping technique for nonlinear single-input discrete-time systems [10] to nonlinear multi-input discrete-time systems. Following the approach [3] and [4], we then design asymptotically convergent nonlinear discrete-time observers for the Euler approximate model and output feedback SPA stabilizing controllers for ships. We give a numerical example to illustrate the design methods. We also summarize the framework for the design of nonlinear sampled-data systems by the Euler approximate model proposed in [6], [11], and [12] and we give the extended result of the integrator backstepping method given in [10] to nonlinear multi-input discrete-time systems in the Appendix.

Notations: Let $\mathbf{N}, \mathbf{R}$ and $\mathbf{R}_{>0}$ be the sets of natural numbers, real numbers and nonnegative real numbers, respectively. Let $\mathbf{C}^{-}=\{\lambda=\alpha+i \beta \mid \alpha<0\}$ and $\mathbf{D}=\{\lambda=\alpha+$ $\left.i \beta \mid \sqrt{\alpha^{2}+\beta^{2}}<1\right\}$. Let $\sigma(M)$ be the set of all eigenvalues of a square matrix $M$. Let $\|x\|$ be the norm of a vector $x$ given by $\|x\|=\sqrt{x^{T} x}$. A function $\alpha$ is of class $K$ if it is continuous, zero at zero and strictly increasing. It is of class $K_{\infty}$ if it is of class $K$ and unbounded. A function $\beta$ : $\mathbf{R}_{\geq 0} \times \mathbf{R}_{\geq 0} \rightarrow \mathbf{R}_{\geq 0}$ is of class $K L$ if for any fixed $t \geq 0$, the function $\beta(\cdot, t)$ is of class $K$ and for each fixed $s \geq 0$ the function $\beta(s, \cdot)$ is deceasing to zero as its argument tends to infinity [5].

\section{NONLINEAR SAMPLED-DATA CONTROL FOR SHIPS}

In this section, we introduce a mathematical model of a ship for the dynamic positioning (DP) problems and its Euler approximate model. We apply Theorems A.1, A.2, and Remark A.1 in the Appendix to design state feedback SPA stabilizing controllers for a ship. Following the approach [3] and [4], we then design observers for the Euler approximate model. Combining the designed observers and state feedback SPA stabilizing controllers, we design output feedback SPA stabilizing controllers.

We first introduce the following notations to describe the equation of motion of a ship. Let $n, e$, and $\psi$ be the North and the East positions of a ship and the yaw angle (orientation) of a ship, respectively, in the Earth-fixed coordinate system and let $\mu, v$ and $r$ be linear velocities in surge, sway and the angular velocity in yaw, i.e., $r=\dot{\psi}$, respectively, decomposed 


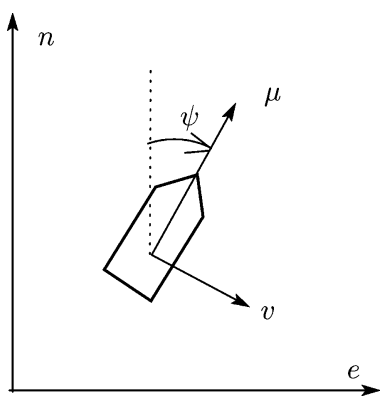

Fig. 1. Coordinate systems.

in the body-fixed coordinate system. Let $\eta_{c}=\left[\begin{array}{lll}n & e & \psi\end{array}\right]^{T}$ and $\nu_{c}=\left[\begin{array}{lll}\mu & v & r\end{array}\right]^{T}$ (see Fig. 1).

In DP problems, the speed of a ship is quite small ( $\mu \simeq 0$, $v \simeq 0, r \simeq 0$ ) and we can assume that the damping forces are linear [3]. Hence, the equation of motion of a dynamically positioned ship can be written as

$$
\dot{\eta}_{c}=R(\psi(t)) \nu_{c}(t), \dot{\nu}_{c}=A \nu_{c}(t)+B u_{c}(t)
$$

where $A=-M^{-1} D, B=M^{-1}$, and

$$
R(\psi)=\left[\begin{array}{ccc}
\cos \psi & -\sin \psi & 0 \\
\sin \psi & \cos \psi & 0 \\
0 & 0 & 1
\end{array}\right]
$$

is the rotation matrix in yaw, $M$ is the inertia matrix including hydrodynamic added inertia, $D$ is the damping matrix and the control forces and moment $u_{c}=\left[\begin{array}{lll}u_{1} & u_{2} & u_{3}\end{array}\right]^{T}$ are provided by thrusters. Note that $R^{-1}(\psi)=R^{T}(\psi)$ and $R(\psi)$ is bounded for any $\psi$.

Let $T$ be a sampling period, $\eta(k)=\eta_{c}(k T)$ and $\nu(k)=$ $\nu_{c}(k T)$. If we set $u_{c}(t)=u(k), t \in[k T,(k+1) T)$, then the exact discrete-time model of (1) is given by

$$
\begin{aligned}
x(k+1) & =x(k)+\int_{k T}^{(k+1) T}\left[\begin{array}{c}
R(\psi(r)) \nu_{c}(r) \\
A \nu(r)+B u(k)
\end{array}\right] d r \\
& =F_{T}^{e}(x(k), u(k))
\end{aligned}
$$

and the Euler approximate model of (1) is given by

$$
\begin{aligned}
& \eta(k+1)=\eta(k)+T R(\psi(k)) \nu(k) \\
& \nu(k+1)=A_{d} \nu(k)+B_{d} u(k)
\end{aligned}
$$

where $x=\left[\begin{array}{ll}\eta^{T} & \nu^{T}\end{array}\right]^{T}, A_{d}=I+T A, B_{d}=T B$, and $\psi(k)=$ $\psi(k T)$ again to avoid the complexity of notations. Since $R(\psi)$ is bounded for any $\psi$ and $A, B$ are constant matrices, it is obvious that $F_{T}^{e}(x, u)$ is locally Lipschitz in $(x, u)$ for sufficiently small $T>0$. Hence, by Remark A.1, if we can find an SPA stabilizing pair $\left(u_{T}, V_{T}\right)$ for (2), then the control input $u_{T}$, given by the designed controllers, SPA stabilizes the nonlinear sampled-data system (1) with $u_{c}(t)=u_{T}(k), t \in[k T,(k+1) T)$ in the continuous-time sense for sufficiently small $T>0$. Here we assume that the sampling period $T>0$ is a design parameter and can be assigned arbitrarily.
In the following sections, using (2), we shall design SPA stabilizing controllers for (1) with $u_{c}(t)=u(k), t \in[k T,(k+$ 1)T) in the continuous-time sense. For this purpose, we assume the following:

A1) $\eta(k)$ and $\nu(k)$ are available to the controller;

A2) $A$ is stable, i.e., $\sigma(A) \subset \mathbf{C}^{-}$.

A1 is needed to apply state feedback controllers and $\mathbf{A 2}$ is a technical assumption to design observers of the form (9) for a ship, but some small size or middle size ships satisfy this assumption [3]. In the case that $\mathbf{A 2}$ is not satisfied, another design method of observers for continuous-time model of a ship is given in [16]. In the rest of this section, we set $L=\operatorname{diag}\left\{l_{1}, l_{2}, l_{3}\right\}$ and $W_{T}(\eta)=1 / 2 \eta^{T} \eta$, where $l_{i}$ are chosen such that $\left|1+T l_{i}\right|<1$ for $i=1,2,3$.

Using the input transformation $u=B^{-1}\left(u_{a}-A \nu\right)$, the Euler approximate model (2) can be rewritten as

$$
\begin{aligned}
& \eta(k+1)=\eta(k)+T R(\psi(k)) \nu(k) \\
& \nu(k+1)=\nu(k)+T u_{a}(k) .
\end{aligned}
$$

Note that the system (3) and (4) is of the form (27) and (28) with $f=0$ and $g(\cdot)=R(\psi(\cdot))$. It is obvious that a virtual control $\nu(k)=\phi_{T}(\eta(k))=R^{T}(\psi(k)) L \eta(k)$ asymptotically stabilizes the subsystem (3) and $W_{T}(\eta)$ is a corresponding Lyapunov function. Since $\phi_{T}(\eta), W_{T}(\eta)$ satisfy all conditions in Theorem A. 2 and we have $r_{T}^{\phi}(\eta)=(I+T L) \eta, \partial W_{T} / \partial \eta=\eta^{T}$, an SPA stabilizing pair $\left(u_{a T}, V_{T}\right)$ for the system (3) and (4) is given by

$$
\begin{aligned}
u_{a T}(x(k))= & -c\left[\nu(k)-\phi_{T}(\eta(k))\right]-\frac{\Delta \tilde{W}_{T}(x(k))}{T} \\
& +\frac{\Delta \phi_{T}(x(k))}{T}
\end{aligned}
$$

and $V_{T}(x)=W_{T}(\eta)+1 / 2\left\|\nu-\phi_{T}(\eta)\right\|^{2}$, where $c>0$ is arbitrary, $\Delta \phi_{T}(x)=\phi_{T}(\eta+T R(\psi) \nu)-\phi_{T}(\eta), \phi_{T}(\eta(k)+$ $T R(\psi(k)) \nu(k))=R^{T}(\psi(k+1)) \operatorname{L\eta }(k+1)$

$$
\Delta \tilde{W}_{T}(x)= \begin{cases}\frac{\Delta \bar{W}_{T}(x)\left[\nu-\phi_{T}(\eta)\right]}{\left\|\nu-\phi_{T}(\eta)\right\|^{2}}, & \nu \neq \phi_{T}(\eta) \\ T R^{T}(\psi)[\eta+T R(\psi) \nu], & \nu=\phi_{T}(\eta)\end{cases}
$$

and $\Delta \bar{W}_{T}(x)=W_{T}(\eta+T R(\psi) \nu)-W_{T}\left(r_{T}^{\phi}(\eta)\right)$. In this case the closed-loop system (3)-(5) is given by

$$
z(k+1)=\Phi(x(k)) z(k)
$$

where $z=\left[\begin{array}{ll}\eta^{T} & z_{2}^{T}\end{array}\right]^{T}, z_{2}=\nu-\phi_{T}(\eta)$, and

$$
\begin{aligned}
& \Phi(x)=
\end{aligned}
$$

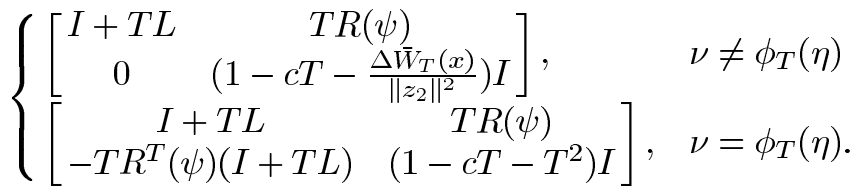

Note also that in this case the condition (23) is rewritten as

$$
z^{T}(k)\left[\Phi^{T}(x(k)) \Phi(x(k))-I\right] z(k) \leq-T \alpha_{3}(\|x(k)\|)+T \delta .
$$

Then by Theorem A.1, we have the following. 
Lemma 2.1: Assume A1. Then there exists $T^{*}>0$ such that for any $T \in\left(0, T^{*}\right)$ the state feedback controller

$$
u_{T}(x(k))=B^{-1}\left[u_{a T}(x(k))-A \nu(k)\right]
$$

SPA stabilizes the system (1) with $u_{c}(t)=u_{T}(x(k)), t \in$ $[k T,(k+1) T)$ in the continuous-time sense.

Since the position of a ship is usually available to the controller in the control problems of a ship, we introduce the sampled observation

$$
y(k)=\eta(k) .
$$

If the assumption $\mathbf{A} \mathbf{2}$ is satisfied, then we can make $\sigma\left(A_{d}\right) \subset$ $\mathbf{D} \backslash\{0\}$ for sufficiently small $T>0$. Then we have the following.

a) There exists $P_{2}>0$ such that $A_{d}^{T} P_{2} A_{d}-P_{2}=-\left(Q_{2}+\right.$ $\hat{Q}_{2}$ ) for any given $Q_{2}, \hat{Q}_{2}>0$.

b) There exists $P_{1}>0$ such that $\left(I-K_{1}\right)^{T} P_{1}\left(I-K_{1}\right)-$ $P_{1}=-\left(Q_{1}+\hat{Q}_{1}\right)$ for any given $Q_{1}, \hat{Q}_{1}>0$ and $K_{1}=$ $\operatorname{diag}\left\{k_{1}, k_{2}, k_{3}\right\}$ with $0<k_{i}<2, i=1,2,3$.

We first design asymptotically convergent nonlinear discretetime observers for the Euler approximate model (2) and (8).

Lemma 2.2: Assume A2. If we choose $K_{1}=$ $\operatorname{diag}\left\{k_{1}, k_{2}, k_{3}\right\}$ with $0<k_{i}<2, i=1,2,3$, positive definite matrices $P_{1}$ and $P_{2}$ satisfying a) and b), respectively, $K_{2}(k)=T P_{2}^{-1} A_{d}^{-T} R^{T}(\psi(k)) P_{1}\left(I-K_{1}\right)$ and $T>0$ is sufficiently small, then

$$
\begin{aligned}
\hat{x}(k+1)= & {\left[\begin{array}{cc}
I & T R(\psi(k)) \\
0 & A_{d}
\end{array}\right] \hat{x}(k)+\left[\begin{array}{c}
0 \\
B_{d}
\end{array}\right] u(k) } \\
& +\left[\begin{array}{c}
K_{1} \\
K_{2}(k)
\end{array}\right]\{y(k)-\hat{\eta}(k)\}
\end{aligned}
$$

is an observer of the system (2) and (8) where $\hat{x}=\left[\begin{array}{ll}\hat{\eta}^{T} & \hat{\nu}^{T}\end{array}\right]^{T}$.

Proofs of Lemma 2.2 and the following theorem are given in the Appendix. Combining the observer (9) and a state feedback controller $u_{T}(\hat{x})$ that is a part of the SPA stabilizing pair for the system

$$
\begin{aligned}
& \hat{\eta}(k+1)=\hat{\eta}(k)+T R(\psi(k)) \hat{\nu}(k) \\
& \hat{\nu}(k+1)=A_{d} \hat{\nu}(k)+B_{d} u(k)
\end{aligned}
$$

we shall design observer-based output feedback SPA stabilizing controllers for the exact discrete-time model. Hence by Theorem A.1 and Remark A.1 we have the following.

Theorem 2.1: Assume $\mathbf{A 2}$ and the conditions in Lemma 2.2. Then there exists $T^{*}>0$ such that for any $T \in\left(0, T^{*}\right)$ the output feedback controller (9) and

$$
\begin{aligned}
u_{T}(\hat{x}(k))= & B^{-1}\left[\bar{u}_{a T}(\hat{x}(k))-A \hat{\nu}(k)\right] \\
\bar{u}_{a T}(\hat{x}(k))= & -c\left[\hat{\nu}(k)-\phi_{T}(\hat{\eta}(k))\right]-\frac{\Delta \tilde{W}_{T}(\hat{x}(k))}{T} \\
& +\frac{\Delta \bar{\phi}_{T}(\hat{x}(k))}{T} .
\end{aligned}
$$

SPA stabilizes the system (1) and (8) with $u_{c}(t)=u_{T}(\hat{x}(k))$, $t \in[k T,(k+1) T)$ in the continuous-time sense where $c>0$ is arbitrary, $\Delta \bar{\phi}_{T}(\hat{x}(k))=\bar{\phi}_{T}(k+1)-\phi_{T}(\hat{\eta}(k))$, $\phi_{T}(\hat{\eta}(k))=R(\psi(k)) L \hat{\eta}(k), \bar{\phi}_{T}(k+1)=R^{T}(\hat{\psi}(k+$ 1)) $L\left[\hat{\eta}(k)+T R^{T}(\psi(k)) \hat{\nu}(k)\right]$

$$
\Delta \tilde{W}_{T}(\hat{x})= \begin{cases}\frac{\Delta \bar{W}_{T}(\hat{x})\left[\hat{\nu}-\phi_{T}(\hat{\eta})\right]}{\left\|\hat{\nu}-\phi_{T}(\hat{\eta})\right\|^{2}}, & \hat{\nu} \neq \phi_{T}(\hat{\eta}) \\ T R^{T}(\psi)[\hat{\eta}+T R(\psi) \hat{\nu}], & \hat{\nu}=\phi_{T}(\hat{\eta})\end{cases}
$$

and $\Delta \bar{W}_{T}(\hat{x})=W_{T}(\hat{\eta}+T R(\psi) \hat{\nu})-W_{T}((I+T L) \hat{\eta})$.

\section{NUMERICAL EXAMPLE}

Consider the equation of motion

$$
\dot{\eta}_{c}=R(\psi(t)) \nu_{c}(t), \dot{\nu}_{c}=A \nu_{c}(t)+B u_{c}(t)
$$

for the supply vessel in Example 11.5 in [3], where $A=-M^{-1} D, B=M^{-1}$, the non-dimensional inertia matrix $M$ and the non-dimensional damping matrix $D$ are given by

$$
\begin{aligned}
M & =\left[\begin{array}{ccc}
1.1274 & 0 & 0 \\
0 & 1.8902 & -0.0744 \\
0 & -0.0744 & 0.1278
\end{array}\right] \\
D & =\left[\begin{array}{ccc}
0.0358 & 0 & 0 \\
0 & 0.1183 & -0.0124 \\
0 & -0.0041 & 0.0308
\end{array}\right]
\end{aligned}
$$

respectively, and we assume that the control input $u(t)$ is realized by $u(t)=u(k), t \in[k T,(k+1) T)$. Then we have $\sigma(A)=\{-0.2428,-0.0627,-0.0318\} \subset \mathbf{C}^{-}$and if we set the sampling period $T=200 \mathrm{~ms}$, then we obtain $\sigma\left(A_{d}\right)=$ $\{0.9514,0.9875,0.9936\} \subset \mathbf{D}$.

For the system (13) with $y(k)=\eta_{c}(k T)$, we shall design output feedback SPA stabilizing controllers. We first design an observer for the Euler approximate model of (13). Let $Q_{1}=40 I, \hat{Q}_{1}=10 I, Q_{2}=5 I, \hat{Q}_{2}=4 I$ and $K_{1}=0.5 I$. Then the positive definite matrices $P_{1}$ and $P_{2}$ in Lemma 2.2 are given by $P_{1}=66.6667 I$ and $P_{2}=\operatorname{diag}\left\{710.8186, P_{2 s}\right\}$, $P_{2 s}=\left[\begin{array}{cc}66.667 & -6.1482 \\ -6.1482 & 95.1090\end{array}\right]$, respectively, and the remaining observer gain $K_{2}$ is given by Lemma 2.2. In this case, we can easily check that the condition (14) is satisfied for any $\psi$. We set $L=-\operatorname{diag}\{2,1,2\}$ and $c=0.2$. Then we have $I+T L=\operatorname{diag}\{0.6,0.8,0.6\}$. Since

$$
\begin{aligned}
& Q_{1}-2 a_{3} K^{T}(k) K(k)>Q_{1} \\
& \quad-2 a_{3}\left[K_{1}^{T}\left(I+2 L^{T} L\right) K_{1}+2 K_{2}^{T}(k) K_{2}(k)\right]>\tilde{Q}_{1}
\end{aligned}
$$

for some $\tilde{Q}_{1}>0$ and for any $\psi$ with $a=b=c=1$, the condition (17) is also satisfied for any $\psi$. Hence, we can apply the output feedback controller (9) and (11) to the system (13). Let $\eta_{c}(0)=\left[\begin{array}{lll}-4 & 3 & -\pi / 4\end{array}\right]^{T}$ and $\nu_{c}(0)=0$ be an initial condition of (13) and let $\hat{\eta}(0)=\hat{\nu}(0)=0$ be an initial condition of the designed output feedback controller. In Fig. 2, the solid line expresses the trajectory of the North-East position of the ship and the symbol like a ship expresses the attitude of the ship at every $0.5 \mathrm{~s}$. As we see Fig. 2, the designed output feedback controller stabilizes the ship. 


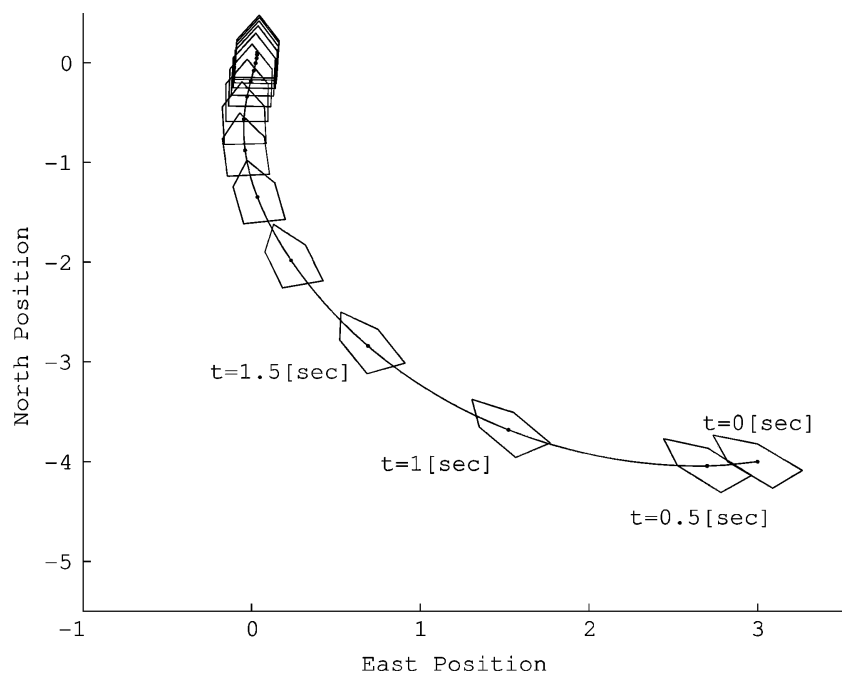

Fig. 2. Trajectory of the North-East position and the attitude of the ship.

We now give a comparison of the designed output feedback SPA stabilizing controllers and the Euler approximation of continuous-time output feedback stabilizing controllers designed by a backstepping technique and a nonlinear observers [3], [5]. The time responses of the yaw angle and the trajectories of the ship are not different so much for both controllers. We can set the sampling period until $T=600 \mathrm{~ms}$ to design the output feedback SPA stabilizing controller (9) and (11). On the other hand, until $T=380 \mathrm{~ms}$ we can find the Euler approximation of continuous-time output feedback stabilizing controllers. Hence there is a possibility that the controller design based on the Euler approximate model makes a sampling period longer than the controller design based on the continuous-time model.

\section{CONClusion}

In this brief, we have considered the nonlinear sampled-data stabilization for dynamically positioned ships. Applying the design methods of nonlinear sampled-data systems proposed in [6], [11], and [12], we have designed SPA stabilizing controllers. We have also given a numerical example to illustrate the design methods. The results in this brief are an extension of the results in [3] and [4] to nonlinear sampled-data systems.

\section{APPENDIX}

Proofs of Lemma 2.2 and Theorem 2.1:

Proof of Lemma 2.2: Let $\tilde{\eta}=\eta-\hat{\eta}, \tilde{\nu}=\nu-\hat{\nu}$ and $\tilde{x}=\left[\begin{array}{ll}\tilde{\eta}^{T} & \tilde{\nu}^{T}\end{array}\right]^{T}$. Then we have

$$
\tilde{x}(k+1)=\left[\begin{array}{cc}
I-K_{1} & T R(\psi(k)) \\
-K_{2}(k) & A_{d}
\end{array}\right] \tilde{x}(k) .
$$

and for the Lyapunov function candidate $V_{o}(k)=$ $1 / 2 \tilde{x}^{T}(k) \operatorname{diag}\left\{P_{1}, P_{2}\right\} \tilde{x}(k)$, we obtain

$$
V_{o}(k+1)-V_{o}(k)=\frac{1}{2} \tilde{\eta}^{T} H_{1} \tilde{\eta}+\frac{1}{2} \tilde{\nu}^{T} H_{2} \tilde{\nu}+\tilde{\nu}^{T} H_{12} \tilde{\eta} .
$$

where $H_{1}=\left(I-K_{1}\right)^{T} P_{1}\left(I-K_{1}\right)-P_{1}+K_{2}^{T}(k) P_{2} K_{2}(k)$, $H_{12}=T R^{T}(\psi(k)) P_{1}\left(I-K_{1}\right)-A_{d}^{T} P_{2} K_{2}(k)$ and $H_{2}=$ $A_{d}^{T} P_{2} A_{d}-P_{2}+T^{2} R^{T}(\psi(k)) P_{1} R(\psi(k))$. Here we shall show that $H_{1} \leq-Q_{1}, H_{2} \leq-Q_{2}$ and $H_{12}=0$. By the choice of $K_{2}, \bar{H}_{12}=0$ is obvious and by the properties of a) and b), we have $H_{1} \leq-Q_{1}-\hat{Q}_{1}+K_{2}^{T}(k) P_{2} K_{2}(k)$ and $H_{2} \leq$ $-Q_{2}-\hat{Q}_{2}+T^{2} R^{T}(\psi(k)) P_{1} R(\psi(k))$. Since we can choose $T>0$ sufficiently small such that

$$
K_{2}^{T} P_{2} K_{2} \leq \hat{Q}_{1}, \quad T^{2} R^{T}(\psi) P_{1} R(\psi) \leq \hat{Q}_{2}
$$

we obtain $H_{1} \leq-Q_{1}$ and $H_{2} \leq-Q_{2}$. Consequently an observer (9) achieves the asymptotical convergence of $(\hat{\eta}, \hat{\nu})$ to $(\eta, \nu)$ for sufficiently small $T>0$ and any initial state $x(0)$ and $\hat{x}(0)$.

Proof of Theorem 2.1: By Theorem A.1 and Remark A.1 it is enough to show that $\left(u_{T}, \tilde{V}_{T}\right)$ is an SPA stabilizing pair for the Euler approximate model (2) and (8), where $u_{T}$ is given by the output feedback controller (9) and (11), $\tilde{V}_{T}(k)=$ $V_{T}(\hat{z}(k))+V_{o}(\tilde{x}(k)), V_{T}(\hat{z})=W_{T}(\hat{\eta})+1 / 2 \hat{z}_{2}^{T} \hat{z}_{2}=1 / 2 \hat{z}^{T} \hat{z}$, and $V_{o}(\tilde{x})=1 / 2\left(\tilde{\eta}^{T} P_{1} \tilde{\eta}+\tilde{\nu}^{T} P_{2} \tilde{\nu}\right)$. Let $\hat{z}_{2}=\hat{\nu}-\phi_{T}(\hat{\eta})$ and $\hat{z}=\left[\begin{array}{ll}\hat{\eta}^{T} & \hat{z}_{2}^{T}\end{array}\right]^{T}$. Then the closed-loop system (2) and (8) with (9) and (11) can be written as

$$
\begin{aligned}
& \hat{z}(k+1)=\Phi(\hat{x}(k)) \hat{z}(k)+\Gamma(k) \hat{z}(k)+K(k) \tilde{\eta}(k) \\
& \tilde{x}(k+1)=\left[\begin{array}{cc}
I-K_{1} & T R(\psi(k)) \\
-K_{2}(k) & A_{d}
\end{array}\right] \tilde{x}(k)
\end{aligned}
$$

where $\Phi(\hat{x})$ is given by (7) and

$$
\begin{aligned}
& \Gamma(k)=\hat{B}(k+1) L \hat{C}(k) \\
& \hat{B}(k+1)=\left[\begin{array}{c}
0 \\
R^{T}(\hat{\psi}(k+1))-R^{T}(\psi(k+1))
\end{array}\right] \\
& \hat{C}(k)=\left[\begin{array}{ll}
I+T L & T R(\psi(k))
\end{array}\right] \\
& K(k)=\left[\begin{array}{c}
K_{1} \\
K_{2}(k)-R^{T}(\psi(k+1)) L K_{1}
\end{array}\right] .
\end{aligned}
$$

Then we want to show that the conditions (22)-(25) are satisfied for $\left(u_{T}, \tilde{V}_{T}\right)$. It is obvious that the conditions (22), (24), and (25) are satisfied and hence it is enough to show (23). By Lemma 2.2 we have

$V_{o}(\tilde{x}(k+1))-V_{o}(\tilde{x}(k)) \leq-\frac{1}{2}\left[\tilde{\eta}^{T}(k) Q_{1} \tilde{\eta}(k)+\tilde{\nu}^{T}(k) Q_{2} \tilde{\nu}(k)\right]$.

We also have

$$
\begin{aligned}
V_{T}(\hat{z}(k+1))-V_{T}(\hat{z}(k)) \\
=\frac{1}{2} \hat{z}^{T}(k)\left[\Phi^{T}(k) \Phi(k)-I\right] \hat{z}(k)+\frac{1}{2}\left(\hat{z}^{T} \Gamma^{T} \Gamma \hat{z}\right)(k) \\
\quad+\frac{1}{2}\left(\tilde{\eta}^{T} K^{T} K \tilde{\eta}\right)(k)+\left(\hat{z}^{T} \Phi^{T} \Gamma \hat{z}\right)(k) \\
\quad+\left(\hat{z}^{T} \Gamma^{T} K \tilde{\eta}\right)(k)+\left(\tilde{\eta}^{T} K^{T} \Phi \hat{z}\right)(k) \\
\leq a_{1} \hat{z}^{T}\left[\Phi^{T} \Phi-I\right] \hat{z}+\frac{1}{2}\left(a+\frac{1}{c}\right)\|\hat{z}\|^{2} \\
\quad+\frac{1}{2}\left(1+\frac{1}{a}+b\right) \hat{z}^{T} \Gamma^{T} \Gamma \hat{z}+a_{3} \tilde{\eta}^{T} K^{T} K \tilde{\eta}
\end{aligned}
$$

for any $a, b, c>0$ where $a_{1}=1 / 2(1+a+1 / c), a_{3}=$ $1 / 2(1+1 / b+c)$ and we have used

$$
2 x^{T} N^{T} M y \leq a x^{T} N^{T} N x+\frac{1}{a} y^{T} M^{T} M y,{ }^{\forall} a>0
$$


in the first inequality. Here for simplicity of notations, we have written $\Phi(k)=\Phi(\hat{x}(k))$ and we have omitted $k$ from the first inequality. Since $R(\psi)$ is bounded for any $\psi$, there exists $m>0$ such that $1 / 2(1+1 / a+b) \hat{z}^{T} \Gamma^{T} \Gamma \hat{z} \leq m\|\hat{z}\|^{2}$ and we have

$$
\begin{aligned}
& V_{T}(\hat{z}(k+1))-V_{T}(\hat{z}(k)) \\
& \quad \leq a_{1} \hat{z}^{T}(k)\left[\Phi^{T}(k) \Phi(k)-I\right] \hat{z}(k)+a_{2}\|\hat{z}(k)\|^{2} \\
& \quad+a_{3} \tilde{\eta}^{T}(k) K^{T}(k) K(k) \tilde{\eta}(k)
\end{aligned}
$$

where $a_{2}=1 / 2(a+1 / c)+m$. Hence we have

$$
\begin{aligned}
& \tilde{V}_{T}(k+1)-\tilde{V}_{T}(k) \\
& \leq a_{1} \hat{z}^{T}(k)\left[\Phi^{T}(k) \Phi(k)-I\right] \hat{z}(k)+a_{2}\|\hat{z}(k)\|^{2} \\
& \quad-\frac{1}{2} \tilde{\eta}^{T}(k)\left[Q_{1}-2 a_{3} K^{T}(k) K(k)\right] \tilde{\eta}(k) \\
& \quad-\frac{1}{2} \tilde{\nu}^{T}(k) Q_{2} \tilde{\nu}(k) .
\end{aligned}
$$

By the choice of $K_{1}$ and $K_{2}(k)$, we can choose $Q_{1}>0$ such that

$$
Q_{1}-2 a_{3} K^{T}(k) K(k)>\tilde{Q}_{1}
$$

for sufficiently small $T>0$ and for some $\tilde{Q}_{1}>0$. Hence we have

$$
\begin{aligned}
\tilde{V}_{T}(k+1)-\tilde{V}_{T}(k) & \leq a_{1} \hat{z}^{T}(k)\left[\Phi^{T}(k) \Phi(k)-I\right] \hat{z}(k) \\
& +a_{2}\|\hat{z}(k)\|^{2}-\frac{1}{2} \tilde{x}^{T}(k)\left[\begin{array}{cc}
\tilde{Q}_{1} & 0 \\
0 & Q_{2}
\end{array}\right] \tilde{x}(k) .
\end{aligned}
$$

Since $\left(u_{T}(\hat{x}), V_{T}(\hat{x})\right)$ is an SPA stabilizing pair for the system (10), there exists $\alpha_{3} \in$ class $K_{\infty}$ such that for any strictly positive real numbers $(\Delta, \delta)$ there exists $T^{*}>0$ such that for all $\hat{z}$ with $\|\hat{z}\|<\Delta$ and $T \in\left(0, T^{*}\right)$

$$
\frac{1}{2} \hat{z}^{T}\left[\Phi^{T}(k) \Phi(k)-I\right] \hat{z} \leq-T \alpha_{3}(\|\hat{z}\|)+T \delta .
$$

Then we have

$$
\begin{aligned}
\tilde{V}_{T}(k+1)-\tilde{V}_{T}(k) \leq & -2 a_{1} T \alpha_{3}(\|\hat{z}(k)\|)+2 a_{1} T \delta \\
& +a_{2} \Delta^{2}-\frac{1}{2} \tilde{x}^{T}(k) \\
& \times\left[\begin{array}{cc}
\tilde{Q}_{1} & 0 \\
0 & Q_{2}
\end{array}\right] \tilde{x}(k) \\
= & -T \hat{\alpha}_{3}\left(\left\|\left[\begin{array}{c}
\hat{z} \\
\tilde{x}
\end{array}\right](k)\right\|\right)+T \hat{\delta}
\end{aligned}
$$

where

$$
\begin{aligned}
\hat{\alpha}_{3}\left(\left\|\left[\begin{array}{c}
\hat{z} \\
\tilde{x}
\end{array}\right]\right\|\right)= & 2 a_{1} \alpha_{3}(\|\hat{z}\|) \\
& +\frac{1}{2 T} \lambda_{\min }\left(\left[\begin{array}{cc}
\tilde{Q}_{1} & 0 \\
0 & Q_{2}
\end{array}\right]\right)\|\tilde{x}\|^{2} \\
\hat{\delta}= & 2 a_{1} \delta+\frac{a_{2}}{T} \Delta^{2}
\end{aligned}
$$

and $\lambda_{\min }(M)$ is the minimum eigenvalue of a matrix $M$. Since $\hat{\alpha}_{3} \in \operatorname{class} K_{\infty}$, we obtain (23).

The Framework for the Design of Nonlinear Sampled-Data Systems: Here we summarize the framework for the design of nonlinear sampled-data systems by Euler approximate model given in [6], [10]-[12].
Consider the nonlinear continuous-time system

$$
\dot{x}=f(x, u), \quad x(0)=x_{0}
$$

where $x \in \mathbf{R}^{n}$ is the state, $u \in \mathbf{R}^{m}$ is the control input realized through a zero-order hold, i.e., $u(t)=u(k),{ }^{\forall} t \in[k T,(k+$ 1) $T$ ) and $T>0$ is a sampling period. Here we assume that for each initial condition and each constant control, there exists a unique solution of (18) defined on some bounded interval of the form $[0, \tau)$. We also assume that the sampling period is a design parameter and can be assigned arbitrarily.

The difference equations corresponding to the exact discretetime model and the Euler approximate model of (18) are denoted by

$$
\begin{aligned}
x(k+1) & =x(k)+\int_{k T}^{(k+1) T} f(x(s), u(k)) d s \\
& =F_{T}^{e}(x(k), u(k)) \\
x(k+1) & =x(k)+T f(x(k), u(k)) \\
& =F_{T}^{E u l e r}(x(k), u(k))
\end{aligned}
$$

respectively, where $x(k)=x(k T)$ again to avoid the complexity of notations. To define semiglobal practical asymptotic (SPA) stability and SPA stability Lyapunov function, we consider the following discrete-time system

$$
x(k+1)=F_{T}\left(x(k), u_{T}(x(k))\right) .
$$

Definition A.1: The system (21) is semiglobal practical asymptotical (SPA) stable if there exists $\beta \in$ class $K L$ such that for any strictly positive real numbers $(D, d)$, there exists $T^{*}>0$ such that for all $T \in\left(0, T^{*}\right)$ and all initial state $x(0)$ with $\|x(0)\| \leq D$, the solution of (21) satisfies $\|x(k)\| \leq$ $\beta(\|x(0)\|, k T)+d$.

Definition A.2: Let $\hat{T}>0$ be given and for each $T \in(0, \hat{T})$ let function $V_{T}: \mathbf{R}^{n} \rightarrow \mathbf{R}_{>0}$ and $u_{T}: \mathbf{R}^{n} \rightarrow \mathbf{R}^{m}$ be defined. Then $\left(u_{T}, V_{T}\right)$ is called an SPA stabilizing pair for $F_{T}$ if there exist $\alpha_{1}, \alpha_{2}, \alpha_{3} \in \operatorname{class} K_{\infty}$ such that for any strictly positive real numbers $(\Delta, \delta)$ there exist strictly positive real numbers $\left(T^{*}, L, M\right)$ with $T^{*}<\hat{T}$ such that for all $x, z \in \mathbf{R}^{n}$ with $\max \{\|x\|,\|z\|\} \leq \Delta$ and $T \in\left(0, T^{*}\right)$

$$
\begin{aligned}
\alpha_{1}(\|x\|) & \leq V_{T}(x) \leq \alpha_{2}(\|x\|) \\
V_{T}\left(F_{T}\left(x, u_{T}(x)\right)\right)-V_{T}(x) & \leq-T \alpha_{3}(\|x\|)+T \delta \\
\left|V_{T}(x)-V_{T}(z)\right| & \leq L\|x-z\| \\
\left\|u_{T}\right\| & \leq M .
\end{aligned}
$$

Theorem A.l: If $\left(u_{T}, V_{T}\right)$ is an SPA stabilizing pair for $F_{T}^{\text {Euler }}$, then $u_{T}$ SPA stabilizes $F_{T}^{e}$.

Remark A.1:

1) If $F_{T}^{e}$ is locally Lipschitz, then there exists $T^{*}>0$ such that for any $T \in\left(0, T^{*}\right) u_{T}$ which SPA stabilizes $F_{T}^{e}$, SPA stabilizes (18) in the continuous-time sense, i.e., there exists $\beta \in$ class $K L$ such that for any strictly positive real numbers $(D, d)$ the solution of the closed-loop system

$$
\dot{x}=f\left(x, u_{T}(x(k))\right), \quad{ }^{\forall} t \in[k T,(k+1) T)
$$


satisfies $\|x(t)\| \leq \beta(\|x(0)\|, t)+d$ for all initial state $x(0)$ with $\|x(0)\| \leq D[6],[12]$.

2) If $F_{T}^{e}$ is locally Lipschitz, then there exists $T^{*}>0$ such that for any $T \in\left(0, T^{*}\right) u_{T}$ which is part of an SPA stabilizing pair $\left(u_{T}, V_{T}\right)$ for $F_{T}^{\text {Euler }}$, SPA stabilizes (18) in the continuous-time sense.

Integrator Backstepping: Consider the nonlinear system of a following strict feedback form

$$
\dot{x}_{1}=f\left(x_{1}\right)+g\left(x_{1}\right) x_{2}, \quad \dot{x}_{2}=u
$$

where $x_{1} \in \mathbf{R}^{n}, x_{2} \in \mathbf{R}^{m}, f(0)=0, f, g$ are differentiable sufficiently many times and the control input $u(t)$ is realized through a zero-order hold. The Euler approximate model of (26) is given by

$$
\begin{aligned}
x_{1}(k+1) & =x_{1}(k)+T\left[f\left(x_{1}(k)\right)+g\left(x_{1}(k)\right) x_{2}(k)\right] \\
& =r_{T}(x(k)) \\
x_{2}(k+1) & =x_{2}(k)+T u(k)
\end{aligned}
$$

where $x=\left[\begin{array}{ll}x_{1}^{T} & x_{2}^{T}\end{array}\right]^{T}$. The next theorem is an extension of the result in [10] to multi-input nonlinear discrete-time systems of the form (27) and (28).

Theorem A.2: Assume that there exist $\hat{T}>0$ and $\left(\phi_{T}, W_{T}\right)$ that is defined for each $T \in(0, \hat{T})$ is a SPA stabilizing pair for the subsystem (27) with a virtual control $x_{2}$. Suppose

1) $\phi_{T}$ and $W_{T}$ are continuously differentiable for any $T \in$ $(0, \hat{T})$.

2) There exists $\tilde{\varphi} \in \operatorname{class} K_{\infty}$ such that $\left\|\phi_{T}\left(x_{1}\right)\right\| \leq \tilde{\varphi}\left(\left\|x_{1}\right\|\right)$ for all $x_{1}$ and $T \in(0, \hat{T})$.

3) For any $\tilde{\Delta}>0$ there exist strictly positive numbers $(\tilde{T}, \tilde{M})$ such that for each $T \in(0, \tilde{T})$ and $\left\|x_{1}\right\| \leq \tilde{\Delta}$ we have $\max \left\{\left\|\partial W_{T} / \partial x_{1}\right\|,\left\|\partial \phi_{T} / \partial x_{1}\right\|\right\} \leq \tilde{M}$.

Then there exists an SPA stabilizing pair $\left(u_{T}, V_{T}\right)$ for (27) and (28). In particular, we can take

$$
\begin{aligned}
u_{T}(x(k))= & -c\left[x_{2}(k)-\phi_{T}\left(x_{1}(k)\right)\right] \\
& -\frac{\Delta \tilde{W}_{T}(x(k))}{T}+\frac{\Delta \phi_{T}(x(k))}{T} \\
V_{T}(x(k))= & W_{T}\left(x_{1}(k)\right)+\frac{1}{2}\left\|x_{2}(k)-\phi_{T}\left(x_{1}(k)\right)\right\|^{2}
\end{aligned}
$$

where $c>0$ is arbitrary, $\Delta \phi_{T}(x)=\phi_{T}\left(r_{T}(x)\right)-\phi_{T}\left(x_{1}\right)$, $r_{T}^{\phi}\left(x_{1}\right)=x_{1}+T\left[f\left(x_{1}\right)+g\left(x_{1}\right) \phi_{T}\left(x_{1}\right)\right]$

$$
\Delta \tilde{W}_{T}(x)= \begin{cases}\frac{\Delta \bar{W}_{T}(x)\left[x_{2}-\phi_{T}\left(x_{1}\right)\right]}{\left\|x_{2}-\phi_{T}\left(x_{1}\right)\right\|^{2}}, & x_{2} \neq \phi_{T}\left(x_{1}\right) \\ T g^{T}\left(x_{1}\right)\left(\frac{\partial W_{T}}{\partial x_{1}}\right)^{T}\left(r_{T}(x)\right), & x_{2}=\phi_{T}\left(x_{1}\right)\end{cases}
$$

and $\Delta \bar{W}_{T}(x)=W_{T}\left(r_{T}(x)\right)-W_{T}\left(r_{T}^{\phi}\left(x_{1}\right)\right)$.
Proof: It is enough to show that $\left(u_{T}(x), V_{T}(x)\right)$ given by (29) and (30) satisfies (22)-(25) in Definition A.2. By Proposition 1 in [10] and the equivalence of $p$-norms, we can show that $V_{T}(x)$ satisfies (22). Similar to the proof of Theorem 2 in [10] (24) and (25) are satisfied. Using the relation $\Delta \bar{W}_{T}=$ $\left[x_{2}-\phi_{T}\left(x_{1}\right)\right]^{T} \Delta \tilde{W}_{T}$ and the Mean Value Theorem, we can also show (23) similar to the proof of Theorem 2 in [10].

\section{ACKNOWLEDGMENT}

The author would like to thank the referees for their useful comments on this brief.

\section{REFERENCES}

[1] M. Arcak and D. Nesic, "A framework for observer design for sampleddata nonlinear systems," Automatica, vol. 40, pp. 1931-1998, 2004

[2] T. Chen and B. A. Francis, Optimal Sampled-Data Control Systems. New York: Springer, 1995.

[3] T. I. Fossen, Marine Control Systems. Trondheim, Norway: Marine Cybernetics, 2002.

[4] T. I. Fossen and A. Grovlen, "Nonlinear output feedback control of dynamically positioned ships using vectorial observerbackstepping," IEEE Trans. Control Syst. Technol., vol. 6, no. 1, pp. 121-128, Jan. 1998.

[5] H. K. Khalil, Nonlinear Systems. Englewood Cliffs, NJ: PrenticeHall, 2002.

[6] D. S. Laila, D. Nesic, and A. Astolfi, "Sampled-data control of nonlinear systems," in Advanced Topics in Control Systems Theory: Lecture Notes From FAP 2005, Lecture Notes in Control and Information Sciences, A. Loria, F.L. Lagarrigue, and E. Panteley, Eds. New York: Springer, 2005, vol. 328, pp. 91-137.

[7] A. Loria, T. I. Fossen, and E. Panteley, "A separation principle for Dynamic positioning of ships: Theoretical and experimental results," IEEE Trans. Control Syst. Technol., vol. 8, no. 2, pp. 332-343, Mar. 2000.

[8] D. Nesic and L. Grune, "Lyapunov based continuous-time nonlinear controller redesign for sampled-data implementation," Automatica, vol. 41, pp. 1143-1156, 2005.

[9] D. Nesic and L. Grune, "Receding horizon control approach to sampled-data implementation of continuous-time controller," Syst. Control Lett., vol. 55, pp. 660-672, 2006.

[10] D. Nesic and A. R. Teel, "Stabilization of sampled-data nonlinear systems via backstepping on their Euler approximate model," Automatica, vol. 42, pp. 1801-1808, 2006.

[11] D. Nesic, A. R. Teel, and P. V. Kokotovic, "Sufficient conditions for stabilization of sampled-data nonlinear systems via discrete-time approximation," Syst. Control Lett., vol. 38, pp. 259-270, 1999.

[12] D. Nesic, A. R. Teel, and E. D. Sontag, "Formulas relating KL stability estimates of discrete-time and sampled-data nonlinear systems," Syst. Control Lett., vol. 38, pp. 49-60, 1999.

[13] T. Perez, Ship Motion Control. New York: Springer, 2005.

[14] K. Y. Pettersen and H. Nijmeijer, "Output feedback tracking control for ships," in New Directions in Nonlinear Observer Design, H. Nijmeijer and T. I. Fossen, Eds. New York: Springer, 1999, pp. 311-331.

[15] K. Y. Pettersen and T. I. Fossen, "Underactuated dynamic positioning of a ship-Experimental results," IEEE Trans. Control Syst. Technol., vol. 8, no. 5, pp. 856-863, Sep. 2000.

[16] A. Robertsson and R. Johansson, "Comments on nonlinear output feedback control of dynamically positioned ships using vectorial observer backstepping," IEEE Trans. Control Syst. Technol., vol. 6, no. 3, pp. 439-441, May 1998. 